\title{
Towards the sequential assimilation of SAR-derived water stages into hydraulic models using the Particle Filter: proof of concept
}

\author{
P. Matgen ${ }^{1,4}$, M. Montanari ${ }^{1}$, R. Hostache ${ }^{1}$, L. Pfister ${ }^{1}$, L. Hoffmann ${ }^{1}$, D. Plaza ${ }^{2}$, V. R. N. Pauwels ${ }^{2}$, G. J. M. De \\ Lannoy $^{2}$, R. De Keyser ${ }^{3}$, and H. H. G. Savenije ${ }^{4}$ \\ ${ }^{1}$ Centre de Recherche Public - Gabriel Lippmann, Département Environnement et Agro-biotechnologies, \\ Belvaux, Luxembourg \\ ${ }^{2}$ Laboratory of Hydrology and Water Management, Ghent University, Ghent, Belgium \\ ${ }^{3}$ Department of Electrical Energy - Systems and Automation, Ghent University, Ghent, Belgium \\ ${ }^{4}$ Water Resources Section, Faculty of Civil Engineering and Geosciences, Delft University of Technology, \\ GA Delft, The Netherlands
}

Received: 23 February 2010 - Published in Hydrol. Earth Syst. Sci. Discuss.: 9 March 2010

Revised: 12 August 2010 - Accepted: 31 August 2010 - Published: 27 September 2010

\begin{abstract}
With the onset of new satellite radar constellations (e.g. Sentinel-1) and advances in computational science (e.g. grid computing) enabling the supply and processing of multimission satellite data at a temporal frequency that is compatible with real-time flood forecasting requirements, this study presents a new concept for the sequential assimilation of Synthetic Aperture Radar (SAR)-derived water stages into coupled hydrologic-hydraulic models. The proposed methodology consists of adjusting storages and fluxes simulated by a coupled hydrologic-hydraulic model using a Particle Filterbased data assimilation scheme. Synthetic observations of water levels, representing satellite measurements, are assimilated into the coupled model in order to investigate the performance of the proposed assimilation scheme as a function of both accuracy and frequency of water level observations. The use of the Particle Filter provides flexibility regarding the form of the probability densities of both model simulations and remote sensing observations. We illustrate the potential of the proposed methodology using a twin experiment over a widely studied river reach located in the Grand-Duchy of Luxembourg. The study demonstrates that the Particle Filter algorithm leads to significant uncertainty reduction of water level and discharge at the time step of assimilation. However, updating the storages of the model only improves the model forecast over a very short time horizon. A more effective way of updating thus consists in adjusting both states and inputs. The proposed methodology, which consists in updat-
\end{abstract}

Correspondence to: P. Matgen

(matgen@lippmann.lu) ing the biased forcing of the hydraulic model using information on model errors that is inferred from satellite observations, enables persistent model improvement. The present schedule of satellite radar missions is such that it is likely that there will be continuity for SAR-based operational water management services. This research contributes to evolve reactive flood management into systematic or quasi-systematic SAR-based flood monitoring services.

\section{Introduction}

An appropriate, rapid, and effective response to any floodinduced disaster is essential. Remote sensing with its extensive spatial coverage in conjunction with prediction models has the potential to deliver the kind and amount of information needed to meet these objectives, especially in data sparse regions across the globe. Optical imagery has been successfully used in the past to extract flooded areas (e.g. Marcus and Fonstad, 2008). However, the rapid flood recession in small- to medium-sized catchments and the typical weather conditions during flood events hamper systematic and global flood detection with visible satellite imagery. With its ability to acquire data during all meteorological conditions, day and night, and its capability to provide information about the extent of open water bodies, Synthetic Aperture Radar (SAR) instruments enable monitoring flood extents over large areas and thus show high potential for facilitating effective flood disaster management. Although significant improvements related to the usefulness, reliability and availability of microwave remote sensing data have been achieved over the

Published by Copernicus Publications on behalf of the European Geosciences Union. 
last few years, there is a clear need for the development of new strategies that allow for a more efficient use of remote sensing-based flood hazard monitoring.

To date, the use of radar imagery is very often limited to an instantaneous binary segmentation into flooded and nonflooded pixels. Additionally, flooded areas retrieved from remote sensing observations have been successfully used a posteriori for calibrating and evaluating hydraulic models (Aronica et al., 2002; Horritt and Bates, 2002, Werner et al., 2005; Pappenberger et al., 2007; Di Baldassarre et al., 2009). Over the last decade many studies have investigated ways to introduce the vertical dimension in flood mapping based on remote sensing observations. Surface water storage can be estimated via the retrieval of inundated areas and water surface elevation. Remote sensing-based techniques thus enable the monitoring of changes in water volume in ways that are not possible using stream gauges (Alsdorf and Lettenmaier, 2003). Two main techniques may be distinguished: (1) direct measuring techniques such as those from the proposed swath altimetry "Surface Water and Ocean Topography" (SWOT) mission (Alsdorf et al., 2007) and (2) indirect measuring techniques based on the estimation of river stages at the land-water interface using topographic maps or Digital Elevation Models (DEM) (e.g. Smith, 1997; Brakenridge et al., 1998; Raclot, 2006; Schumann et al., 2007a; Matgen et al., 2007a). The reliability and repeatability are the main advantages associated with water level products stemming from wide swath altimetry. The expected accuracy is $0.5 \mathrm{~m}$. However, the technique can only provide surface water storage fluctuations in large rivers (50-100 $\mathrm{m}$ width) at a sub-monthly time scale. Higher sampling rates are achievable through a combination of indirect water level measurements obtained from various high-resolution SAR sensors. The accuracy of the data (ranging from $0.2-3 \mathrm{~m}$ ) is unknown a priori and depends on many factors (e.g. topography, sensor characteristics, DEM). Maps of water stage can be expected to contain more information than binary wet/dry maps and would thus be able to constrain the uncertainty in hydraulic models more efficiently than binary patterns (Schumann et al., 2009). The calibration of flood inundation models with remote sensing-derived water level data has been investigated in only a few studies in the recent past (Schumann et al., 2007b; Hostache et al., 2009; Mason et al., 2009).

Even if the prediction models are thoroughly calibrated with observations stemming, for instance, from remote sensing observations, a mismatch will remain between modelled and observed state and flux data. Hydraulic models do provide spatially and temporally continuous surface fields but they are subject to uncertainties in input data, model structure, model parameters and initial conditions. Periodically updating the models with observations may thus reduce the predictive uncertainty of the models (Bates et al., 2004). This approach relies on the rationale that the merging of model results and remote sensing observations, both subject to considerable uncertainty, yields the best possible model analyses and eventually better predictions. Schumann et al. (2009) argue that the lack of maturity of processing chains needed to extract hydraulically relevant information from remote sensing data explains why, to date, only point measurements of river stage and discharge are routinely assimilated in hydraulic models (Madsen and Skotner, 2005; Neal et al., 2007; Smith et al., 2009). Data assimilation studies investigating the usefulness of remote sensing derived water level measurements are rare.

In one of the few data assimilation studies using realevent satellite data (as opposed to synthetic satellite observations), Neal et al. (2009) showed that it is possible to retrieve discharge and level estimates from ENVISAT Advanced Synthetic Aperture Radar (ASAR) imagery when combined with hydraulic modelling using an Ensemble Kalman Filter (EnKF). In their proof-of-concept study the assimilation of SAR-derived water level data led to a significant reduction in discharge and water level uncertainty over that derived from a sequence of atmospheric, hydrologic and hydraulic models alone. In another study dealing with real-event satellite data, Matgen et al. (2007b) used a direct insertion method that forced the prognostic state (i.e. water stage) of the model to fall within the confidence interval of ENVISAT and European Remote Sensing Satellite (ERS) SAR-derived water stages. Although the modelled water levels were more accurate immediately after assimilation, during subsequent time steps the modelled water surface line gradually bounced back to the initial water surface line obtained without data assimilation. Several hours after image acquisition the results obtained with and without assimilation completely overlapped. The effect of the assimilation of remote sensing information is thus limited by the persistence of the initial condition and due to the dominating effect of the upstream boundary condition (i.e. inflows); only a temporary improvement can be achieved through a mere re-initialization of hydraulic models (Schumann et al., 2009). Andreadis et al. (2007) came to a similar conclusion in their proof-of-concept study. To tackle the problem of non-persistent model improvements, the authors successfully exploit the time correlation of model errors. By complementing the state updating approach with an update of the boundary condition, thereby correcting errors in forcing data as part of the data assimilation scheme, they achieve a more sustainable model improvement. Hostache et al. (2009) propose a parameter updating approach as an alternative to the state updating method that was used in previous studies. A variational data assimilation method using distributed water level information was used to combine in an optimal sense measurement data and a 2-D shallow water model. The remote sensing-derived water level data allowed identifying clusters of optimal Manning friction coefficients for various areas in the floodplain that are characterized by homogeneous friction. Parameter updating has a significant history with respect to the assimilation of in situ hydrometric measurements. A detailed review can be found in Smith et al. (2008). However, as it was pointed out by 


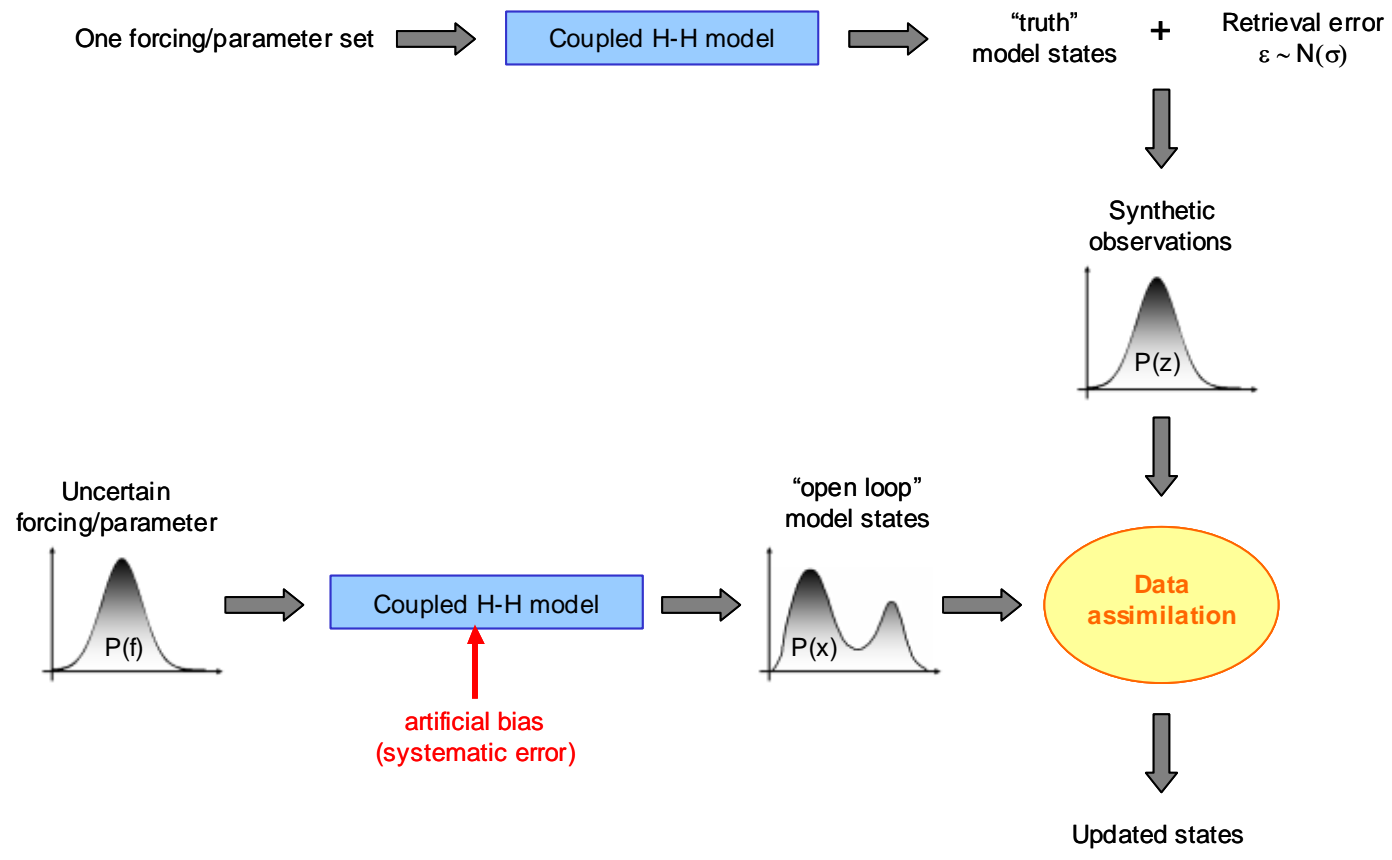

Fig. 1. Scheme of the data assimilation experiment (adapted after Moradkhani et al., 2008). H-H model stands for coupled hydrologichydraulic model.

Kirchner (2006), sequential state updating is conceptually preferable to sequential parameter updating because parameter updating violates a basic principle of physically-based modelling, namely that the constants should stay constant while variables vary.

As pointed out by Schumann et al. (2009), there is no doubt that a comprehensive remote sensing data assimilation framework has the potential of becoming a critical component in future flood forecasting systems. The objective of this paper is to contribute to the ongoing debate on how to integrate remote sensing-derived data into hydraulic modelling. In particular, we aim at investigating the accuracy and sampling rate requirements for remote sensing inferred water stage products. Moreover, we want to propose a data assimilation framework that is adapted to the form of probability density functions that are typically associated with modelled and remotely sensed water stages. Lastly, we need to define new ways for updating the forcings in order to achieve a persistent improvement of hydraulic models with the information contained in satellite observations.

\section{Methods}

\subsection{Experimental design}

Figure 1 shows the synthetic experiment design used in this study for assessing the added-value of the proposed data assimilation technique.
The adopted experimental design is similar to the ones presented by Andreadis et al. (2007) and Moradkhani et al. (2008). The experiment consists in assimilating synthetic water stage observations into an ensemble of hydraulic models whose upstream boundary conditions (flow hydrographs) are produced using corrupted semi-distributed hydrologic models. De Lannoy et al. (2007) showed that one of the main underlying assumptions of filtering schemes, namely that both observations and model predictions are unbiased, is often not satisfied. For this reason they recommend that data assimilation schemes should include a bias correction algorithm. While we assume remote sensing observations to be unbiased and to only contain a random error component, we consider model predictions to contain both random and systematic errors. A two-step approach is therefore adopted to generate model predictions. Step one consists in randomly disturbing model parameters, forcings and initial conditions of the hydrologic model. The procedure for generating the unbiased ensemble of model realizations is explained in detail in Sect. 3.4. Step two consists in introducing an artificial bias to the simulated upstream boundary discharges and to run the hydraulic model with these biased forcings (i.e. the "open loop" simulations). It is important to note that the same hydraulic model (same model structure, initial conditions and parameter sets) is used for all coupled hydrologichydraulic model runs.

Synthetic observations are generated by adding white noise to a so-called "truth" model. The "truth" corresponds to one hydraulic model realization which is integrated for 
one upstream inflow. The latter corresponds to one unbiased hydrologic model realization. The "truth" and the ensemble of model predictions are generated separately but using the same model structure. Only the model parameters, forcings and initial conditions of the hydrologic model component differ. Here we assume that there is no structural error. Furthermore, it is worth mentioning that in the context of a proof-of-concept study, the "truth" does not attempt to precisely represent the real system, but only a hypothetical system, which approaches the real world. For a given time step, the "truth" thus corresponds to a water surface line that is computed between the upstream and downstream boundaries of the river reach. The prognostic states of the model consist of water levels that are computed for every river cross section (1-D) or grid point (2-D/3-D) of the model domain. As a matter of fact, synthetic observations can be easily mapped to modelled state variables. In order to produce temporally and spatially distributed synthetic observations of water stage, the "truth" is corrupted by a white noise. Hence, we assume that there is no non-stationary bias and variance in the observational error structure. Different observation perturbation magnitudes are chosen in order to investigate the performance of the assimilation scheme as a function of observation uncertainty. Reported root mean squared errors of remote sensing-derived water levels range from $20 \mathrm{~cm}$ (Schumann et al., 2008) to $3 \mathrm{~m}$ (Oberstadler et al., 1997) depending mostly on satellite sensors, floodplain topography and DEM accuracy. It is worth mentioning here that the upcoming SWOT mission (Alsdorf et al., 2007) is expected to deliver water elevation products with an accuracy of $50 \mathrm{~cm}$ (Andreadis et al., 2007). Similarly, the impact of the sampling rate of satellite data will be investigated by changing the time interval between observations. Investigated observation frequencies range between 12 and $48 \mathrm{~h}$. While no single satellite provides water stage data with standard deviations down to $20 \mathrm{~cm}$ every $12 \mathrm{~h}$, the combination of high-precision data sets stemming from different highresolution satellite missions does provide coverage over Europe and many other part of the world in less than 2 days. Alternatively, coarse resolution imagery with associated revisit times of $48 \mathrm{~h}$ and less might be considered. However, it has to be noted that because of a strong inverse relationship between spatial resolution and revisit times of satellites, higher observation uncertainties need to assumed in this case (e.g. 1-2 m).

\subsection{Data assimilation algorithm}

The data assimilation technique implemented in thi s study is based on the Particle Filter (PF), an ensemble filtering method that has its origin in Bayesian estimation. Unlike the widely used EnKF (Evensen, 1994; Burgers et al., 1998), which simplifies the recursive estimation by assuming a Gaussian distribution of the observation and model errors, the PF relaxes the need for restrictive assumptions regarding the forms of the probability density functions; that is, $\mathrm{PF}$ can easily manage the propagation of a non-Gaussian distribution through nonlinear hydrologic and hydraulic models (Moradkhani, 2008). A detailed description of the PF can be found in Moradkhani (2005a). A short description is given here.

Early implementations of the PF were based on the Sequential Importance Sampling (SIS) method, which is a Sequential Monte Carlo procedure developed mainly to allow approaching the probability distributions of state variables via a number of independent random samples, called particles. These particles are sampled directly from the statespace to represent the posterior probability, and a weight is computed for each particle according to the information contained in the observations. Next, an estimate is computed based on these particles and weights. Weights and estimates are sequentially updated every time an observation becomes available.

The SIS algorithm has serious limitations that need to be addressed. The particles tend toward dispersion owing to the stochastic behaviour of the system, with the result that many of them drift away from the "truth" and obtain negligible weight. Only a few particles do participate effectively in the filter causing also wastage of computation resources. To reduce the degeneracy of the SIS method, a selection (i.e. resampling) stage needs to be introduced to eliminate samples with low weight and replicate samples with high weight. The most common resampling scheme is the Sequential Importance Resampling (SIR) developed by Gordon et al. (1993). The authors refer to Moradkhani et al. (2005a) and Weerts et al. (2006) for more detailed explanations of the PF and its use in hydrologic sciences.

Each particle represents the water surface line resulting from one hydraulic model at the assimilation time $t_{k}$. The differences between particles stem from differences in upstream hydrographs that were propagated across the hydraulic model. The number of prognostic state variables (i.e. water levels) corresponds to the number of cross sections or grid points. Since observations are direct measurements of the state, the mapping of observations to modelled state variables is straightforward. One weight per particle and per model output location is computed by using a Gaussian likelihood, as follows:

$w_{k}^{i, j}=p\left(z_{k}^{j} \mid x_{k}^{i, j}\right)=\frac{1}{\sigma_{k}^{j} \sqrt{2 \pi}} e^{\frac{-\left(z_{k}^{j}-h\left(x_{k}^{i, j}, \vartheta^{i}\right)\right)}{2 \sigma_{k}^{j} 2}}$

In Eq. (1), $w_{k}^{i, j}$ is the weight (probability) of $i$-th particle and $j$-th observation at $k$-th discrete time step (assimilation time), $z$ is the observation vector (i.e. synthetic water stages), $x$ is the state vector (i.e. prior estimates of water stages), $h$ is a nonlinear operator that relates state and observation (in this case $h(x, \boldsymbol{\theta})=x), \boldsymbol{\theta}$ is a vector of time-invariant parameters and $\sigma$ is the standard deviation associated to the observation. The proposed weighting procedure (Eq. 1) assumes 
water stage observations to be normally distributed. A more likely theoretical distribution function has not been specified in previous studies. In one of the rare studies dealing with real-event data, Schumann (2007) showed that probability density functions of ENVISAT ASAR-derived water stage observations are non Gaussian at many cross-sections. However, the spatial coverage offered by remote sensing means that it is not necessary to consider all measurements derived from an image. Therefore, Neal et al. (2009) propose a subsampling method that only assimilates measurements that did not fail a normality test.

It is worth mentioning that the weighting procedure (Eq. 1) can be easily adapted to any kind of empirical or theoretical distribution function. In fact, weights can be attributed to every simulated water stage based on the empirical histogram or theoretical pdf of remote sensing-derived water levels. Moreover, Schumann et al. (2007) demonstrated that the observational error standard deviation $\sigma$ related to SAR-derived water stages is characterized by spatial variability. For sake of simplicity, the assumption is made here that $\sigma$ gemains constant along the length of the river reach. Similarly, we assume that the observational error standard deviation $\sigma$ is the same at every assimilation time.

Subsequently, one single weight per particle is computed by applying the joint probability theory for independent variables (Eq. 2), which is then normalized (Eq. 3). $N_{p}$ is the number of particles and $N_{o}$ is the number of observations that is equal to the number of model output locations.

$$
\begin{gathered}
w_{k}^{i}=\prod_{j=1}^{N_{o}} w_{k}^{i, j} \\
W_{k}^{i}=\frac{w_{k}^{i}}{\sum_{i=1}^{N_{p}} w_{k}^{i}}
\end{gathered}
$$

The probability obtained at the previous steps allows for computing an expectation of the updated water stage as follows:

$$
E\left(x_{k}\right)=\sum_{i=1}^{N_{p}} x_{k}^{i} W_{k}^{i}
$$

The above-described procedure represents the SIS algorithm and provides the posterior pdf of the particles $\left\{x_{k}^{i}, W_{k}^{i}\right\}$ and the expectation $E\left(x_{k}\right)$. However, as explained previously, an additional resampling step is needed in order to avoid degeneracy of the system toward a few particles. The SIR algorithm proposed by Gordon et al. (1993) replicates the particles in proportion to their weights: those with an associated low importance weight are replaced with replicas of those having higher weight. Finally, the same weight $\frac{1}{N_{p}}$ is assigned to each "new" particle in order to create equally weighted random measures $\left\{x_{k}^{i}, \frac{1}{N_{p}}\right\}$.
As a result, at $k$-th discrete time step, each model will be re-initialized using updated water levels before being integrated until the next assimilation time. The re-initialization corresponds to the substitution of water levels in the model. The SIR algorithm has been used throughout the study. It is important to note that the SIR algorithm also suffers from particle degeneracy. According to Smith et al. (2008), the resampling step only helps reducing the degeneracy of the particles. Moreover, another problem that may arise, termed "sample impoverishment", causes particles with high weights to be selected many times leading to a loss of diversity in the sample. In fact, due to the discrete approximation of the filtering density, inaccuracies accumulate over many time steps and generate a clustering of particles in small areas of the state-space (Fearnhead, 2002). In the experimental set-up that is used in this study, the problem of sample impoverishment is partially avoided through a loose coupling of the hydrologic and hydraulic models. Only water levels are resampled, while the spread in the forcings (i.e. hydrographs at the upstream boundary) is maintained. This means that in case only a few particles are replicated, the diversity in the forcings causes a direct divergence of the replicated state variables (i.e. water levels).

\subsection{Coupled hydrologic-hydraulic model}

The modelling sequence consists of the loose coupling of a semi-distributed hydrologic model and a 1-D hydraulic model. The sequence consists of the discharge hydrographs computed by the hydrologic model being integrated with the hydraulic model as upstream boundary condition. The upstream boundary discharge is produced using the Community Land Model (CLM) (Dai et al., 2003), a global land surface model built on the fundaments of ecological climatology, over the $356 \mathrm{~km}^{2}$ drainage area of the Alzette River extending upstream of the gauging station at Pfaffenthal. The model's structure is characterized by a grid limited to 4 cells. The surface heterogeneity is represented in the surface data using different fractions of land cover type and different plant functional types through patches. The observed atmospheric forcings were assumed to be uniform and kept constant for the different patches for all the grid cells. Ten soil physical parameters from CLM were estimated for the observed discharge. The selected parameter set is directly related to surface runoff and baseflow processes (for a detailed description of the parameters and physical processes in CLM 2.0, the reader is referred to De Lannoy, 2006). For the modelled discharge, a linear routing model was applied (Troch et al., 1994).

The hydraulic model is implemented over a $19 \mathrm{~km}$ reach of the Alzette River between the gauging stations Pfaffenthal and Mersch. Since in this area the flow direction is mainly parallel to the channel, the 2-D flow field that is typically related to riverbank overtopping can be accurately approximated by a 1-D representation (i.e. velocity components 
in directions other than the main flow direction are not accounted for). Thus, the widely used Hydrologic Engineering Center River Analysis System - HEC-RAS (HEC-RAS 4.0, 2008) - was set-up for 1-D river flow computation. A spatially uniform Manning friction coefficient for both the channel and floodplain was chosen, 0.047 and 0.184 respectively (for further details about the calibration procedure and study area we cross-refer to Montanari et al., 2009). It is worth mentioning that the methodology can also be extended to rivers characterized by a more complex geometry (which need to be modelled 2-D). In this case the prognostic state variables would correspond to the water levels simulated at every grid point. Since the hydrologic and hydraulic models are only loosely coupled, it is also worth mentioning that the hydrologic model could be replaced by any other model.

\subsection{Ensemble generation}

In order to represent the hydraulic model uncertainty, an ensemble of upstream boundary conditions (i.e. discharge) model was generated with CLM2.0.

For this purpose, and following the methodology proposed by Pauwels et al. (2009), the meteorological forcings, the model parameters and the initial conditions were disturbed by adding a Gaussian random number to their deterministic values. The standard deviation of this random number was set to a fraction of the parameter value. Obviously, it is important to realistically assess the model uncertainty in order to conduct the assimilation study in a meaningful way (Moradkhani et al., 2005b). The parameters need to be perturbed in such a way that the spread of the discharge ensemble optimally brackets the range of discharge observations, thereby providing a challenging test case for the assimilation of remote sensing data. A review on procedures to generate meaningful ensembles in hydrologc modelling and data assimilation can be found in Moradkhani et al. (2005b).

It is expected that on average the ensemble mean differs from the observation by a value that is equal to the time average of the ensemble spread (De Lannoy et al., 2006). As a prerequisite for calculating the verification measures, first the ensemble spread $\left(\operatorname{ensp}_{k}\right)$, the ensemble mean square error $\left(\mathrm{mse}_{k}\right.$ ), and the ensemble skill (ensk $k_{k}$ ) need to be computed at each time step $k$ :

$\operatorname{ensp}_{k}=\frac{1}{N_{p}} \sum_{i=1}^{N_{p}}\left(q_{k}^{i \mathrm{f}}-q_{k}^{\mathrm{f}}\right)^{2}$

$\mathrm{mse}_{k}=\sum_{i=1}^{N_{p}}\left(q_{k}^{i \mathrm{f}}-q_{k}^{0}\right)^{2}$

$\operatorname{ensk}_{k}=\left(q_{k}^{\mathrm{f}}-q_{k}^{0}\right)^{2}$

$N$ is the number of particles and the superscripts ${ }^{\mathrm{f}}$ and ${ }^{0}$ refer to the forecast variable and the ensemble mean, respectively. The following verification measures (Eqs. 8 and 9) control that (i) the ratio between the ensemble skill $\left(\mathrm{ensk}_{k}\right)$ and the ensemble spread $\left(\mathrm{ensp}_{k}\right)$ is close to one and that (ii) the truth is statistically indistinguishable from a member of the ensemble (De Lannoy et al., 2006).

$$
\begin{aligned}
& \frac{\langle\text { ensk }\rangle}{\langle\text { ensp }\rangle} \approx 1 \\
& \frac{\langle\sqrt{\text { ensk }}\rangle}{\langle\sqrt{\mathrm{sec}}\rangle} \approx \sqrt{\frac{N+1}{2 N}}
\end{aligned}
$$

In Eqs. (8) and (9), <.> indicates an average over the simulation period.

The optimal fractions of parameter values (i.e. standard deviation of random number added to the deterministic values) were determined using an interval search. An ensemble size of 64 members was used, and the ensemble size with the best match for both statistics was used in the data assimilation study.

The ensemble verification statistics obtained for the CLM with 64 particles are $\frac{\langle\text { ensk }\rangle}{\langle\text { ensp }\rangle}=1.2$ and $\frac{\langle\sqrt{\text { ensk }}\rangle}{\langle\sqrt{\mathrm{mse}}\rangle} \approx \sqrt{\frac{N+1}{2 N}}=$ $0.71 \approx 0.74$.

As mentioned above, these verification measures can be interpreted as indications that the ensemble covers the range of observations. It is important to note here that they are calculated over a 6 month period from 1 January 2007 to 30 June 2007. Figure 2 shows the resulting ensemble for the studied flood event (1 January 15:00-7 January 23:00, 2003). Finally, an artificial positive bias of $25 \%$ has been introduced to the simulated upstream boundary discharge in order to simulate the bias that is inherent in most model realizations, even after calibration. Due to the artificial bias of $25 \%$, the "open loop" water level simulations are significantly larger in comparison to the "truth". Smaller bias values could have been chosen without significantly impacting the results of the study.

The ensemble of hydraulic model realizations, hereafter called "open loop" simulations, has been produced by integrating the hydraulic model core with all the members of the ensemble of discharges generated by the hydrologic model for the analysis period 1 January 15:00-7 January 23:00, 2003. The ensemble of water surface lines represents the coupled H-H model uncertainty, as shown in Fig. 3 for a given time step.

\section{Results}

The hydraulic model has been used to simulate water levels using the ensemble of hydrographs generated by the CLM (Fig. 2), from which water levels at each cross section can be extracted at the time of a virtual satellite overpass. A first test was performed at the time step $t_{24}$ (i.e. $24 \mathrm{~h}$ after the onset of the flood wave) during the rising limb of the flood. Before assimilating the synthetic observations obtained from 


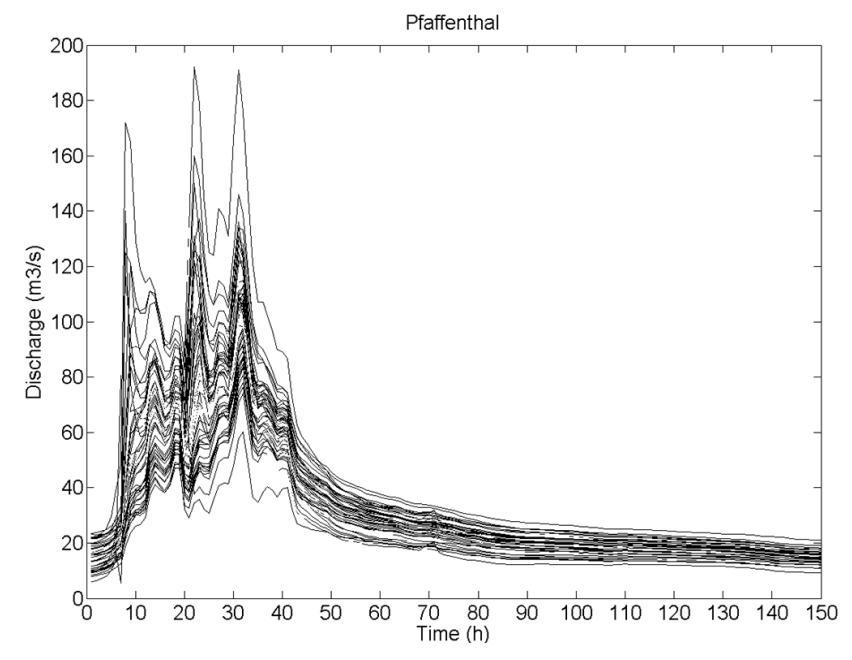

Fig. 2. Ensemble of discharges generated by the CLM.

the "truth" model run, the average standard deviation of simulated variables along the river reach is $0.43 \mathrm{~m}$ at $t_{24}$. By using the SIR algorithm, particles with low weight are replaced by those having higher weight during the analysis step. The weights given to the individual particles are computed based on the information contained in the satellite data (Eq. 1).

First we investigated the effect of observation uncertainty on the mean forecast error and ensemble spread after assimilation. The experiment was repeated for eight sets of observations generated assuming different water stage errors (assumed to be unbiased). Figure 4 shows the histograms of the water stages of the resampled particles corresponding to an intermediate cross section (located about $6.5 \mathrm{~km}$ upstream the downstream boundary) at the assimilation time $t_{24}$. The results were obtained with $10 \mathrm{~cm}, 30 \mathrm{~cm}, 50 \mathrm{~cm}, 70 \mathrm{~cm}, 1 \mathrm{~m}$, $2 \mathrm{~m}, 5 \mathrm{~m}$ and $10 \mathrm{~m}$ observation error standard deviation, respectively. The standard deviation of $30 \mathrm{~cm}$ corresponds to a published value of root mean squared error (RMSE) obtained in the same study area with an ENVISAT ASAR flood image fused with a high resolution high accuracy Lidar DEM (Hostache et al., 2009) and may thus be considered as a realistic accuracy assumption for this experiment. Lower standard deviations might eventually be achieved with high resolution SARs, high resolution DEMs and favourable floodplain geometries. The expected elevation accuracy of the upcoming SWOT mission corresponds to $50 \mathrm{~cm}$ (Andreadis et al., 2007). Higher standard deviation should help our understanding of the usefulness of highly uncertain observations.

As can be seen from the various panels of Fig. 4, the ensemble spread representing forecast uncertainty, decreases and the a posteriori expectation (i.e. mean of the resampled particles) gets closer to the "truth" for smaller standard deviations. For comparison the histogram corresponding to the particles before the resampling (a priori estimate) is plotted.

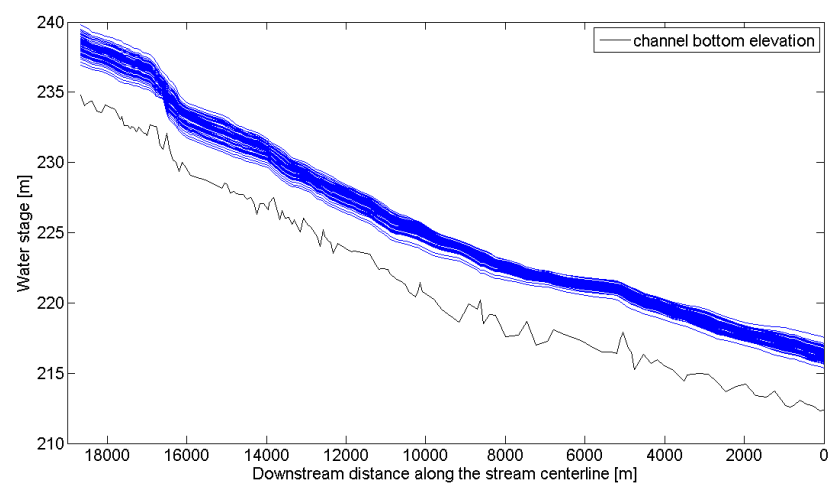

Fig. 3. Ensemble of water surface lines generated by the hydraulic model at time $24 \mathrm{~h}$.

With a $10 \mathrm{~cm}$ standard deviation of observations, the PF allows discarding the majority of the particles and creates many replicas of the particle that is closest to the "truth". We observe that with increasing standard deviation the retained number of particles becomes larger and the ensemble spread gradually extends. However, up until $5 \mathrm{~m}$ of standard deviation the expectation is close to the "truth" which means that the PF removed the artificial positive bias that was added to the model simulations. The distribution of water levels associated with the individual particles is centred around the "truth". However, more and more particles are retained after the resampling step since the discriminatory power of the observations progressively reduces. The rather satisfactory results obtained with perturbation magnitudes up to $5 \mathrm{~m}$ indicate that with errors typical for coarse resolution imagery (Schumann et al., 2010) significant model improvements can be achieved. This result is interesting as observation frequencies of $24 \mathrm{~h}$ and less are systematically achievable only with coarse resolution imagery. This illustrates the potential of coarse resolution imagery for hydrological monitoring and prediction applications. With $10 \mathrm{~m}$ standard deviation, however, the PF does not allow any improvement with respect to the a priori estimate and particles with low weight are taken into account. Similar results were obtained for all the cross sections distributed over the length of the reach (not depicted here). The value of the observations clearly depends on the observation and pre-assimilation forecast error.

Following the resampling step, the model is propagated in time and its result becomes the forecast in the next analysis cycle. To do so, the hydraulic model is re-initialized with updated water levels and integrated until the next analysis cycle (i.e. when new observations become available). Figures 5 and 6 show the stage hydrographs corresponding to the first cross section (upstream boundary) and an intermediate cross section along the river reach. Here we assume an imaging frequency of $12 \mathrm{~h}$ and a standard deviation of unbiased observation errors of $30 \mathrm{~cm}$. Note that observation frequencies of $12 \mathrm{~h}$ and higher can currently only be achieved by combining 

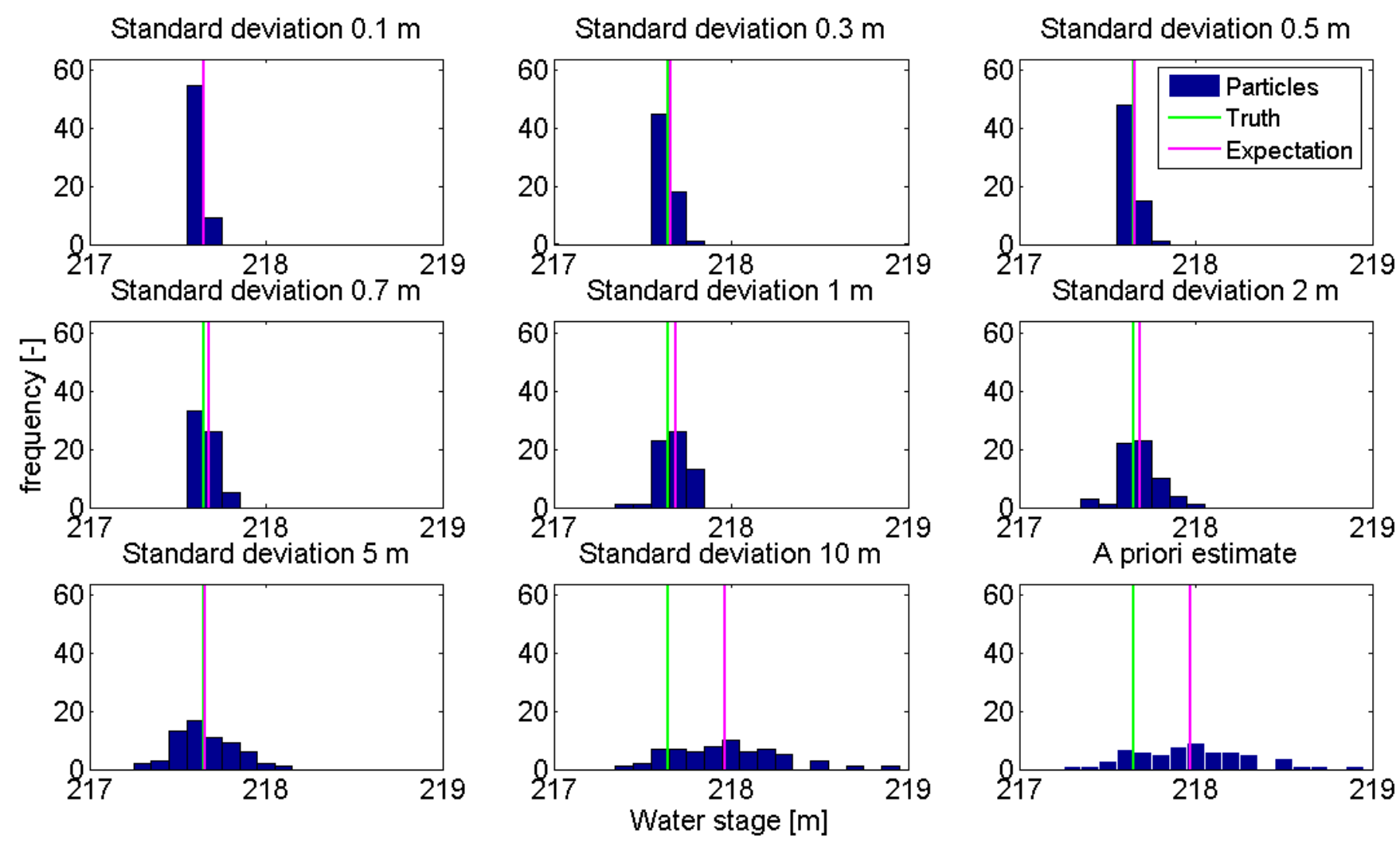

Fig. 4. Histograms of water stages at an intermediate cross section computed with different values of standard deviation of the observations.

data sets from different SAR missions and constellations. The effect of lower sampling rates will be investigated at a later stage.

In both figures, the black lines represent the ensemble of particles corresponding to the model results obtained using the filter. The green line is the "truth", the cyan line is the expectation computed considering the open loop and the magenta line is the expectation computed using the filter. As already observed in the histograms in Fig. 4, during the analysis step the expectation computed with the filter is very close to the "truth", meaning that the PF leads to a significant increase of the accuracy and reduction of the uncertainty of the model. Nevertheless, within a few time steps after the analysis, the forecast returns towards the open loop model realisation. The RMSE is calculated between the expectation and the truth over the entire time window, with the exception of the time steps of the analysis that are not included. Note that the RMSE is practically the same with and without assimilation (Tables 1 and 2). Hence the improvement that was obtained during the analysis step is lost very quickly. The improvement becomes slightly more persistent moving downstream along the river, which is an indication of the dominating effect of the boundary condition in the river reach under investigation. During the experiment, the assimilation time step was changed step by step from $48 \mathrm{~h}$ down to $12 \mathrm{~h}$, as
Table 1. RMSE [m] of the ensemble mean water stage with respect to the "truth", computed at the upstream boundary over the entire time window excluding the analysis steps for $0.3 \mathrm{~m}$ of standard deviation of the observation $(\sigma)$ and various assimilation frequencies $(F)$. At this cross section the RMSE obtained with the mean of the "open loop" simulation equals $0.39 \mathrm{~m}$.

\begin{tabular}{cccc}
\hline $\begin{array}{c}F \rightarrow \\
\sigma \downarrow\end{array}$ & $48 \mathrm{~h}$ & $24 \mathrm{~h}$ & $12 \mathrm{~h}$ \\
\hline $0.3 \mathrm{~m}$ & 0.39 & 0.39 & 0.39 \\
\hline
\end{tabular}

shown in Tables 1 and 2, in order to investigate the performance of the data assimilation scheme as a function of the frequency of the observations. Higher observation frequencies were not considered because they are not plausible with respect to acquisitions of SAR images. Increasing the observation frequency did not lead to any significant improvement with respect to the forecast as demonstrated in Tables 1 and 2.

In Tables 1 and 2 the RMSE is computed by comparing the ensemble mean water stage and the truth over the entire event time window at the two representative river cross sections analysed in Figs. 5 and 6. Albeit the proposed particle 
Table 2. RMSE $[\mathrm{m}]$ of the ensemble mean water stage with respect to the "truth", computed at an intermediate cross section over the entire time window excluding the analysis steps for $0.3 \mathrm{~m}$ of standard deviation of the observation $(\sigma)$ and various assimilation frequencies $(F)$. At this cross section the RMSE obtained with the mean of the "open loop" simulation equals $0.34 \mathrm{~m}$.

\begin{tabular}{cccc}
\hline $\begin{array}{c}F \rightarrow \\
\sigma \downarrow\end{array}$ & $48 \mathrm{~h}$ & $24 \mathrm{~h}$ & $12 \mathrm{~h}$ \\
\hline $0.3 \mathrm{~m}$ & 0.33 & 0.31 & 0.28 \\
\hline
\end{tabular}

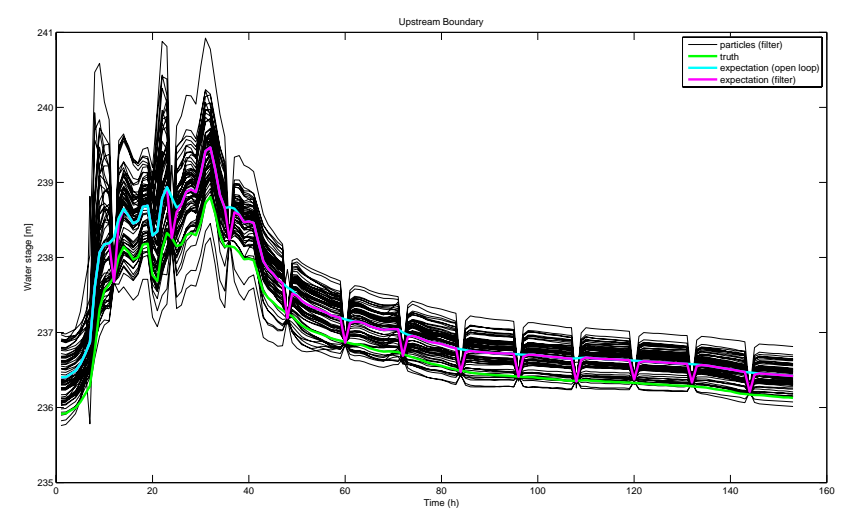

Fig. 5. Stage hydrographs at the upstream boundary with a 12-h assimilation interval.

filter-based assimilation scheme is able to significantly reduce model uncertainty during the analysis step, the improvement completely disappears within a few hours following the assimilation. This result confirms the one obtained by Matgen et al. (2007b) who encountered the same problem in their study on the same river reach. Andreadis et al. (2007) stated that in channels where boundary conditions almost fully govern the flow regime, the time window of model skill improvement due to assimilation of observations is expected to be short. In order to overcome this problem, an enhanced data assimilation scheme is proposed that allows updating the forcing of the hydraulic model using information on model error that is obtained during the analysis step. The estimate of water stage, $E\left(x_{k}\right)$, as computed in Eq. (4), is used to retrieve the corresponding estimate of discharge, $E\left(Q_{k}\right)$ for the upstream boundary using the internal rating curve computed by the hydraulic model Hec-Ras. Then a relative error term is computed at the assimilation time:

$\Delta Q_{k}=\frac{\overline{Q_{k}^{1}}-E\left(Q_{k}^{1}\right)}{\overline{Q_{k}^{1}}}$

$\overline{Q_{k}^{1}}$ is the ensemble discharge average generated by the hydrologic model during the analysis step (Fig. 3) that is the upstream boundary of the hydraulic model (cross section

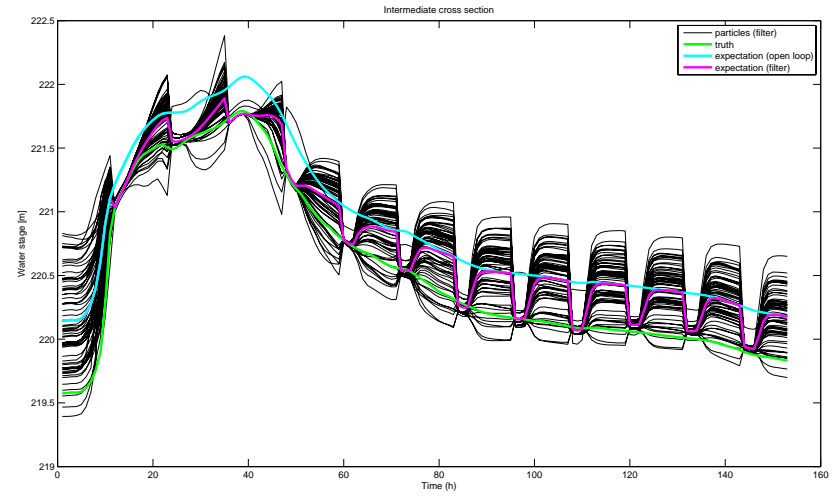

Fig. 6. Stage hydrographs at an intermediate cross section with a 12-h assimilation interval.

number 1 , as indicated by the superscript). Another option consists in computing a relative error term for each particle separately. However, this approach results in the convergence of all particles on the value of the expected discharge. As a matter of fact, the model error at the end of the analysis is assumed to be zero and the spread at subsequent time steps is significantly constrained. To keep some uncertainty around the expectation and to allow the ensemble spread to rapidly return towards the original variance, each particle is updated individually using the same relative error term (Eq. 10).

Then, from time $k=t_{\text {ass }}+1$ until the next assimilation time, every member of the ensemble of discharge hydrographs represented in Fig. 3 is corrected by applying the relative error term $\Delta Q_{k}$ as follows:

$Q_{i}^{1}=Q_{i}^{1}-\Delta Q_{t_{\mathrm{ass}}^{I}} Q_{i}^{1}, i \in\left[\left(t_{\mathrm{ass}}^{I}+1\right) ; t_{\mathrm{ass}}^{I I}\right]$

The method is based on the assumption that relative model errors remain constant and that correcting the inflows by the same relative error term at subsequent time steps will improve the accuracy of the model predictions from time steps $t_{\text {ass }}+1$ through the next assimilation steps. Another approach would be to use an autoregressive model that uses the current value of model error to predict the model errors at subsequent time steps (e.g. Neal et al., 2007). The parameters of such a forecast model would need to be estimated with observed discharge records. However, such data are very often not available. The proposed method, albeit very simple, may be used as an estimate of the propagation of model errors in the absence of discharge data. The underlying assumption is that current model errors are due to an over- or underestimation of water stored in the basin and that if the basin acts like a linear reservoir (as it is often the case, see e.g. Fenicia et al., 2006) the error in discharge decreases following an exponential decline (in the absence of rainfall).

Similar to the first data assimilation scheme, the impact of various imaging frequencies on the analysis and forecast precision has been investigated. Hereafter the experiment 
Table 3. RMSE $[\mathrm{m}]$ of the ensemble mean water stage opposed to the "truth", computed at an intermediate cross section over the entire time window excluding the analysis step for $0.3 \mathrm{~m}$ of standard deviation of the observation $(\sigma)$ and increasing assimilation frequency $(F)$. At this cross section the RMSE obtained with the mean of the "open loop" simulation equals $0.34 \mathrm{~m}$.

\begin{tabular}{cccc}
\hline $\begin{array}{c}F \rightarrow \\
\sigma \downarrow\end{array}$ & $48 \mathrm{~h}$ & $24 \mathrm{~h}$ & $12 \mathrm{~h}$ \\
\hline $0.3 \mathrm{~m}$ & 0.25 & 0.19 & 0.18 \\
\hline
\end{tabular}

performed with an interval of $48 \mathrm{~h}$ between observations is analyzed. Note that the synthetic observations were obtained by adding white noise with a standard deviation of $0.3 \mathrm{~m}$ to the "truth" model.

Figure 7 shows the effect of water stage assimilation when both states (i.e. water levels) and inputs (i.e. inflows) are updated. The proposed approach resolves the issue of non persistent model improvement. However, there is a risk to overcorrect the model with the error forecast model applied in this study. When the assimilation is performed during the rising limb of the hydrograph, the time window of the model improvement is short, whereas when the assimilation is performed during the recession the reduction of the uncertainty is persistent (after the assimilation at $72 \mathrm{~h}$, the assimilation at $120 \mathrm{~h}$ appears to be unnecessary). The explanation of this result can be found in the analysis of the time series of absolute error (truth - expectation). This error is very unstable during the rising limb when new input errors (i.e. error in precipitation) are added at every time step. This input error (i.e. random error) is impossible to predict. During the falling limb, however, the relative model error remains almost constant and can easily be predicted (i.e. systematic error). This explains why the systematic error forecast model that is used for the boundary inflow correction is very efficient during the recession period. The filter is prone to more random error in the rising limb, which eventually results in more uncertain analyses. In this synthetic setup, we know that the chosen bias model is good and that the jumps in the results are entirely due to unexplained random noise, rather than to bias. Figure 8 shows flow hydrographs resulting from an experiment where the time interval between observations is $24 \mathrm{~h}$. With respect to the previous results, it should be noted that increasing the frequency of the assimilations improves the performance of the assimilation system during the rising limb and around the peak, whereas no significant positive effect is obtained during the recession, where a single update appears to be enough.

Similar to Tables 1 and 2, Table 3 shows the RMSE of the ensemble mean water stage as opposed to the "truth". The RMSE only slightly changes between assimilation frequencies of 12 and $24 \mathrm{~h}$, whereas the RMSE changes rather

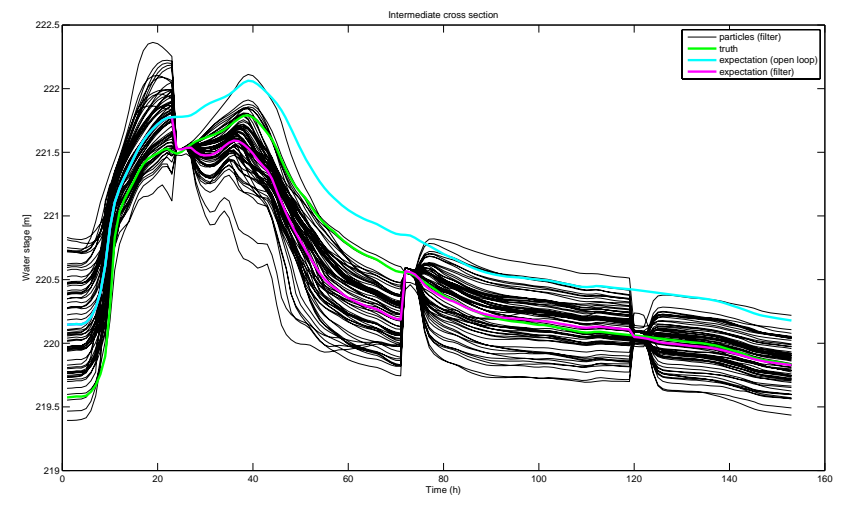

Fig. 7. Stage hydrographs at an intermediate cross section with a 48-h assimilation interval with input bias correction.

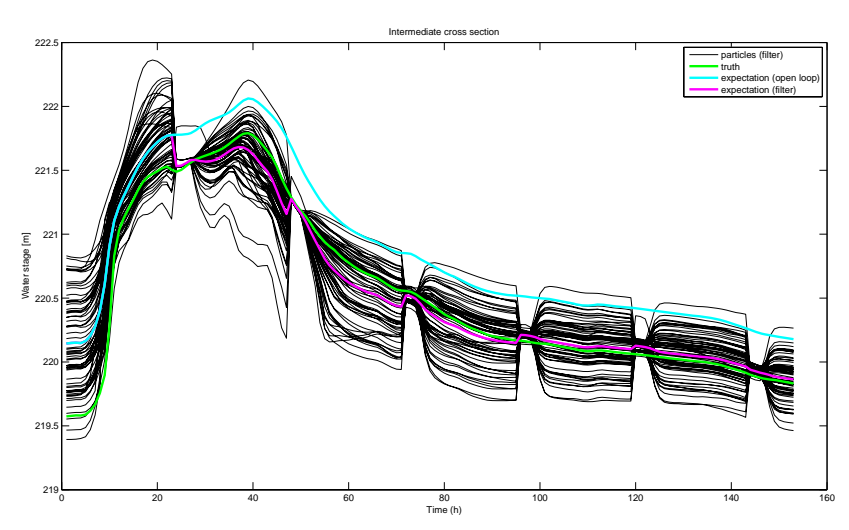

Fig. 8. Stage hydrographs at an intermediate cross section with a 24-h assimilation interval with input bias correction.

significantly when increasing the sampling rate from 24 to $48 \mathrm{~h}$. This is due to the fact that with an assimilation frequency of $48 \mathrm{~h}$, there is no observation available at the onset of the falling limb. Therefore it has to be noted that this result is very specific for the flow hydrograph under investigation. In general, the result demonstrates that the required imaging frequency depends on the temporal correlation of model error. As a matter of fact, during the rising limb the imaging frequency needs to be higher than during the recession. It can also be noticed that the RMSE with assimilation is significantly lower than without.

\section{Discussion and conclusions}

Our study confirms the findings obtained in previous studies: information contained in radar flood images can lead to improved flood inundation modelling (e.g. Bates et al., 2004; Pappenberger et al., 2007; Neal et al., 2009). The experiments conducted with synthetically generated observations integrated with a 1-D hydraulic model further show that: 
1. The Particle Filter enables to correct water depth from a corrupted hydraulic model by assimilating synthetic observations. Significant model improvements could be achieved with observation error standard deviations up to $5 \mathrm{~m}$. This results shows that remote sensing-derived water stage data can be efficiently used to update flood models. The results further indicate that satisfactory results may be obtained with standard deviations that are realistically achievable with coarse resolution imagery. Given the higher sampling rates associated with coarse resolution imagery, this result highlights the potential of this type of imagery for hydrological monitoring and prediction applications.

2. The effectiveness of the filter is not only dependent on the accuracy of the observations but also on the model itself. The latter is represented by the spread of an ensemble of water level simulations. In instrumented catchments, the hydraulic model uncertainty is generally low compared to remote sensing observation uncertainty. With currently available remote sensing-derived water level products the proposed assimilation scheme thus appears to be particularly useful in ungauged catchments, where the use of coupled hydrologic-hydraulic models is required and no training data is available for calibrating sophisticated inflow correction models. This conclusion is well in line with the findings of Neal et al. (2009) in their recent study.

3. Our results further show that it is crucial to make use of the time correlation of model errors in order to significantly and persistently improve the hydraulic model. For this reason, we propose a rather simplistic error forecast model as an essential component of the proposed assimilation methodology. During the analysis step, the expectation of water levels resulting from the assimilation of remote sensing observations into a hydraulic model provides the current value of the model error. The proposed inflow correction model is based on the assumption that model errors are due to a temporary over- or underestimation of the amount of water stored in the basins. In this case, relative model errors remain constant. Groundwater reservoirs in many catchments are known to be linear (e.g. Fenicia et al., 2006). The proposed error forecast model is particularly well suited for predictions in ungauged catchments as no calibration step is required. The experiments show that during the rising limb when rainfall errors continuously add to the model error in an unpredictable way, the imaging frequency needs to be higher than during recession in order to compensate for the shortcomings of the inflow correction model.

4. Our research has clearly demonstrated that merely updating the state variable of the model (water level and hence water storage), only improves the model forecast over a very short time horizon. A much more effective way of updating consists in adjusting the fluxes at the upstream boundaries of the model, which in general have the highest uncertainty as a result of the poorly known rainfall distribution over a catchment, or uncertainty in hydrologic model predictions. By selecting the most likely model runs, the PF, unlike the EnKF that was used in similar studies (e.g. Andreadis et al., 2007), conserves mass for each particle. Potentially, the PFbased assimilation scheme could be used to infer input data (e.g. whole-catchment precipitation) and model parameters that gave the most likely simulations given the observations. The approach may indeed be viewed as a way to diagnose the functioning of hydrologic systems. The usefulness of such an application needs to be investigated in future studies.

This approach was developed in a way that it could be easily transformed into practice. The next key task is to test the methodology with real event data and to investigate problems related to the spatially and temporally variable and potentially biased non Gaussian distribution of water level observations. We expect the Particle Filter to be the preferred filter because it provides flexibility regarding the form of the probability densities of both model simulations and remote sensing observations. The weighting procedure can be easily adapted to any kind of empirical distribution function. Moreover, we feel that more sophisticated methods for boundary inflow correction are needed to further enhance the performance and persistence of the assimilation. Finally, it is important to investigate in future studies the value of remote sensing-derived water level data with respect to hydrometric station data. Given the complementary characteristics of both data sets, the combination of both promises to yield the best overall results. More research is needed on this issue.

As pointed out by Neal et al. (2009) this kind of investigation is timely because in the very near future new constellations of satellite radar missions will lead to a significant increase in the volume of data available for space-borne water level estimation. The expected faster supply and processing of multi-mission satellite data are compatible with real-time crisis management requirements. This study shall thus contribute to the shift from purely reactive space-borne flood monitoring services towards systematic, proactive and global floodplain monitoring services.

Acknowledgements. This study was part of the HYDRASENS project, financed by the National Research Fund (FNR) of the Grand Duchy of Luxembourg and the Belgian Federal Science Policy Office in the framework of the STEREO II research programme (Contract no. SR/00/100). The authors would like to thank the reviewers, in particular Giuliano di Baldessarre, Jeffrey Neal, Paul Smith and Hamid Moradkhani for their valuable contribution to the development of this paper.

Edited by: H. Cloke 


\section{References}

Alsdorf, D. E. and Lettenmaier, D. P.: Tracking fresh water from space, Science, 301, 1485-1488, 2003.

Alsdorf, D. E., Rodriguez, E., and Lettenmaier, D. P.: Measuring surface water from space, Rev. Geophys., 45, RG2002, doi:10.1029/2006RG000197, 2007.

Andreadis, K. M., Clark, E. A., Lettenmaier, D. P., and Alsdorf, D. E.: Prospects for river discharge and depth estimation through assimilation of swath-altimetry into a rasterbased hydrodynamics model, Geophys. Res. Lett., 34, L10403, doi:10.1029/2007GL029721, 2007.

Aronica, G., Bates, P. D., and Horritt, M. S.: Assessing the uncertainty in distributed model predictions using observed binary pattern information within GLUE, Hydrol. Process., 16, 20012016, 2002.

Arya, L. M., Richter, J. C., and Paris, J. F.: Estimating profile water storage from surface zone soil moisture measurements under bare field conditions, Water Resour. Res., 19, 403-412, 1983.

Bates, P. D., Horritt, M. S., Aronica, G., and Beven, K.: Bayesian updating of flood inundation likelihoods conditioned on flood extent data, Hydrol. Process., 18, 3347-3370, 2004.

Brakenridge, G. R., Tracy, B. T., and Knox, J. C.: Orbital SAR remote sensing of a river flood wave, Int. J. Remote Sens., 19, 1439-1445, 1998.

Burgers, G., Leeuwen, P. J. V., and Evensen, G.: Analysis scheme in the ensemble Kalman filter, Mon. Weather Rev., 126, 17191724, 1998.

Dai, Y., Zeng, X., Dickinson, R. E., Baker, I., Bonan, G., Bosilovich, M., Denning, S., Dirmeyer, P., Houser, P., Niu, G., Oleson, K., Schlosser, C. A., and Yang, Z.-L.: The common land model (CLM), B. Am. Meteorol. Soc., 84, 1013-1023, 2003.

De Lannoy, G. J. M., Houser, P. R., Pauwels, V. R. N., and Verhoest, N. E. C.: State and bias estimation for soil moisture profiles by an ensemble Kalman filter: Effect of assimilation depth and frequency, Water Resour. Manag., 43(6), W06401, doi:10.1029/2006WR005100, 2007.

De Lannoy, G. J. M., Houser, P. R., Pauwels, V. R. N., and Verhoest, N. E. C.: Assessment of model uncertainty for soil moisture through ensemble verification, J. Geophys. Res., 111, D10101, doi:10.1029/2005JD006367, 2006.

De Lannoy, G. J. M.: Assimilation of soil moisture observations into a spatially distributed hydrologic model, PhD Dissertation, Ghent University, Belgium, 2006.

Di Baldassarre, G., Schumann, G., and Bates, P. D.: A technique for the calibration of hydraulic models using uncertain satellite observations of flood extent, J. Hydrol., 367, 276-282, 2009.

Evensen, G.: Sequential data assimilation with a nonlinear quasigeostrophic model using Monte Carlo methods to forecast error statistics, Journal of Geophysical Research, 99 (C5), 1014310162, 1994.

Fearnhead, P.: Markov chain Monte Carlo, sufficient statistics and particle filters, J. Comput. Graph. Stat., 11, 848-862, 2002.

Fenicia, F., Savenije, H. H. G., Matgen, P., and Pfister, L.: Is the groundwater reservoir linear? Learning from data in hydrological modelling, Hydrol. Earth Syst. Sci., 10, 139-150, doi:10.5194/hess-10-139-2006, 2006.

Gordon, N., Salmond, D., and Smith, A. F. M.: Novel approach to nonlinear and non-Gaussian Bayesian state estimation, Proceedings of the Institute of Electrical Engineering, Part F, 140,
107-113, 1993.

HEC-RAS 4.0: online available at: http://www.hec.usace.army.mil/ software/hec-ras/documents/HEC-RAS_4.0_Reference_Manual. pdf, last access: 5 May 2008.

Horritt, M. S. and Bates, P.D.: Evaluation of 1-D and 2-D numerical models for predicting river flood inundation, J. Hydrol., 268, 8799, 2002.

Hostache R., Lai, X., Monnier, J., and Puech, C.: Assimilation of spatially distributed water levels into a shallow-water flood model. Part II: use of a remote sensing image of Mosel River, J. Hydrol., under review, 2010.

Hostache, R., Matgen, P., Schumann, G., Puech, C., Hoffmann, L., and Pfister, L.: Water level estimation and reduction of hydraulic model calibration uncertainties using satellite SAR images of floods, IEEE T. Geoscience and Remote Sens., 47, 431441, 2009.

Kirchner, J. W.: Getting the right answers for the right reasons: linking measurements, analyses and models to advance the science of hydrology, Water Resour. Res., 42, 8, doi:10.1029/2005WR004362, 2006.

Madsen, H. and Skotner, C.: Adaptive state updating in real-time river flow forecasting a combined filtering and error forecasting procedure, J. Hydrol., 308, 302-312, 2005.

Marcus, W. A. and Fonstad, M. A.: Optical remote mapping of rivers at sub-meter resolutions and watershed extents, Earth Surf. Proc. Land., 33, 4-24, 2008.

Mason, D. C., Bates, P. D., and Dall'Amico, J. T.: Calibration of uncertain flood inundation models using remotely sensed water levels, J. Hydrol., 368, 224-236, 2009.

Matgen, P., Schumann, G., Henry, J.-B., Hoffmann, L., and Pfister, L.: Integration of SAR-derived inundation areas, high precision topographic data and a river flow model toward real-time flood management, International Journal of Applied Earth Observation and Geoinformation, 9, 247-263, 2007a.

Matgen, P., Schumann, G., Pappenberger, F., and Pfister, L.: Sequential assimilation of remotely sensed water stages in flood inundation models, Remote Sensing for Environmental Monitoring and Change Detection (Proceedings of Symposium HS3007 at IUGG2007, Perugia, July 2007). IAHS Publ. 316, 78-88, 2007 b.

Montanari, M., Hostache, R., Matgen, P., Schumann, G., Pfister, L., and Hoffmann, L.: Calibration and sequential updating of a coupled hydrologic-hydraulic model using remote sensingderived water stages, Hydrol. Earth Syst. Sci., 13, 367-380, doi:10.5194/hess-13-367-2009, 2009.

Moradkhani, H.: Hydrologic Remote Sensing and Land Surface Data Assimilation, Sensors, 8, 2986-3004, doi:10.3390/s8052986, 2008.

Moradkhani, H., Hsu, K.-L., Gupta, H. V., and Sorooshian, S.: Uncertainty assessment of hydrologic model states and parameters: Sequential data assimilation using the particle filter, Water Resour. Res., 41, W05012, doi:10.1029/2004WR003604, 2005a.

Moradkhani, H., Sorooshian, S., Gupta, H. V., and Houser, P. R.: Dual state-parameter estimationof hydrological models using ensemble Kalman filter, Adv. Water Resour., 28, 135-147, 2005b.

Neal, J. C., Atkinson, P. M., and Hutton, C. W.: Flood inundation model updating using an ensemble Kalman filter and spatially distributed measurements, J. Hydrol., 336, 401-415, 2007.

Neal, J., Schumann, G., Bates, P.D., Buytaert, W., Matgen, P., and Pappenberger, F.: A data assimilation approach to discharge from 
space, Hydrol. Process., 23, 3641-3649, 2009.

Pappenberger, F., Frodsham, K., Beven, K., Romanowicz, R., and Matgen, P.: Fuzzy set approach to calibrating distributed flood inundation models using remote sensing observations, Hydrol. Earth Syst. Sci., 11, 739-752, doi:10.5194/hess-11-739-2007, 2007.

Pauwels, V. R. N. and De Lannoy, G. J. M.: Ensemble-based assimilation of discharge into rainfall-runoff models: A comparison of approaches to mapping observational information to state space, Water Resour. Res., 45, W08428, doi:10.1029/2008WR007590, 2009.

Raclot, D.: Remote sensing of water levels on floodplains: a spatial approach guided by hydraulic functioning, International Journal of Remote Sensing, 27, 2553-2574, 2006.

Schumann, G., Bates, P. D., Horritt, M. S., Matgen, P., and Pappenberger, F.: Progress in integration of remote sensing derived flood extent and stage data and hydraulic models, Rev. Geophys, 47, RG4001, doi:10.1029/2008RG000274, 2009.

Schumann, G., Matgen, P., Pappenberger, F., Hostache, R., and Pfister, L.: Deriving distributed roughness values from satellite radar data for flood inundation modelling, J. Hydrol., 344, 96-111, 2007b.

Schumann, G., Matgen, P., Pappenberger, F., Hostache, R., Puech, C., Hoffmann, L., and Pfister, L.: High-resolution 3-D flood information from radar for effective flood hazard management, IEEE T. Geosci. Remote , 45, 1715-1725, 2007a.
Smith, L. C.: Satellite remote sensing of river inundation area, stage, and discharge: a review, Hydrol. Process., 11, 1427-1439, 1997.

Smith, P. J., Hughes, D., Beven, K., Cross, P., Tych, W., Coulson, G., and Blair, G.: Towards the provision of site specific flood warnings using wireless sensor networks, Meteorol. Appl., 16, 57-64, 2009.

Smith, P. J., Beven, K. J., and Tawn, J. A.: Detection of structural inadequacy in process-based hydrological models: a particle-filtering approach, Water Resour. Res., 44, W01410, doi:10.1029/2006WR005205, , 2008.

Troch, P. A., Smith, J. A., Wood, E. F., and De Troch, F. P.: Hydrologic controls of large floods in a small basin: Central Appalachian case study, J. Hydrol., 156, 285-309, 1994.

Weerts, A. H. and El Serafy, G. Y. H.: Particle filtering and ensemble Kalman filtering for state updating with hydrological conceptual rainfall-runoff models, Water Resour. Res., 42, W09403, doi:10.1029/2005WR004093, 2006.

Werner, M., Blazkova, S., and Petr, J.: Spatially distributed observations in constraining inundation modelling uncertainties, Hydrol. Process., 19, 3081-3096, 2005. 